\title{
Determining dominant partial waves in photoproduction
}

\author{
Yannick Wunderlich*广 \\ Helmholtz-Institut für Strahlen- und Kernphysik, University of Bonn \\ E-mail: wunderlich@hiskp.uni-bonn.de
}

Farah N. Afzal

Helmholtz-Institut für Strahlen- und Kernphysik, University of Bonn

E-mail: afzal@hiskp.uni-bonn.de

\section{Annika Thiel}

Helmholtz-Institut für Strahlen- und Kernphysik, University of Bonn

E-mail: thiel@hiskp.uni-bonn.de

\section{Reinhard Beck}

Helmholtz-Institut für Strahlen- und Kernphysik, University of Bonn

E-mail: beck@hiskp.uni-bonn.de

Important contributions to the study of the excitation spectra of baryons are provided by measurements of polarization observables in reactions that involve particles with spin. Pseudoscalar meson photoproduction poses an example-reaction that has been under intense investigation recently.

The extraction of resonance-parameters from the polarization-data in so-called energy-dependent fits, often involving sophisticated reaction- theories and analyzing several reaction-channels at once, represents the state-of-the-art method to get to the spectrum. While yielding important scientific insights, the construction and handling of such models is very sophisticated.

The analysis of partial waves at single energies in so-called energy-independent fits represents a simpler problem. However, due to mathematical ambiguities rising exponentially in number with the amount of non-zero partial waves, such analyses still require some experience. In order to obtain first insights into a newly measured polarization dataset, simpler alternatives are desirable. Here we present the very basic method of moment-analysis for pseudoscalar meson photoproduction, which proceeds by analyzing just the angular distributions. The order of the dominant partial waves contributing in the data can be extracted quickly using this method. Furthermore, the coefficients extracted from the angular distributions show interesting composition-patterns in terms of multipoles and allow for instructive comparisons to models.

This work was supported by the Deutsche Forschungsgemeinschaft (SFB/TR16).

XVII International Conference on Hadron Spectroscopy and Structure - Hadron2017

25-29 September, 2017

University of Salamanca, Salamanca, Spain

\footnotetext{
* Speaker.

${ }^{\dagger}$ The speaker wishes to thank the organizers for the wonderful conference.
} 
In hadron spectroscopy, for instance baryon spectroscopy, resonance parameters are typically extracted from scattering data by means of so-called energy-dependent (ED) fits. Here, a reaction-theory is constructed taking into account coupled-channel effects, which should satisfy well-established theoretical principles (analyticity, unitarity and crossing). Once such a model has been fitted to data, resonances are extracted as poles in the complex energy plane. The BonnGatchina model [1] is a well-known example for such an analysis.

Another, technically less sophisticated, approach to analyze scattering observables consists of single-energy (SE) fits, or truncated partial-wave analyses (TPWAs). As an example, consider a $2 \rightarrow$ 2-reaction with spinless particles. The only observable is the differential cross section $\sigma_{0}$, which, up to phase-space factors which are suppressed here, is given in terms of the amplitude as $\sigma_{0}=|A(W, \theta)|^{2}$. The infinite partial wave series reads $A(W, \theta)=\sum_{\ell=0}^{\infty}(2 \ell+1) A_{\ell} P_{\ell}(\cos \theta)$. In case this series is truncated at some maximal angular momentum quantum number $\ell_{\max }$ and then inserted into the cross section, one obtains the expansion (see reference [2])

$$
\sigma_{0}(W, \theta)=\frac{q}{k} \sum_{n=0}^{2 \ell_{\max }} a_{n}^{\sigma_{0}}(W) P_{n}(\cos \theta), \quad a_{n}^{\sigma_{0}}(W)=\sum_{\ell, k=0}^{\ell_{\max }} A_{\ell}^{*}(W) \mathscr{C}_{\ell k}^{n} A_{k}(W) .
$$

Then, in a SE fit, one would try to solve for the real- and imaginary parts of (possibly) phaseconstrained partial waves. The extraction of Legendre-moments using the first equation in (1) is still simple and numerically well-behaved. However, the bilinear equations in the second relation of equation (1) generally admit multiple discrete ambiguities [3] and thus generally require additional (model-) input in order to be solved. One could also try to be content just with the moment-expansion of the angular distribution and try to learn as much as possible about the data using just this expansion.

The same spirit, i.e. trying to learn as much as possible about a particular dataset using the simple Ansatz of moment-analysis, may be applied to more complicated reactions, for instance the photoproduction of a single pseudoscalar meson. One may consider the most common case of pion photoproduction, $\gamma N \longrightarrow \pi N$, but the following formulae hold even with other pseudoscalar mesons in the final state.

Photoproduction is described model-independently using 4 complex spin-amplitudes, for instance CGLN-amplitudes $\left\{F_{i}(W, \theta), i=1, \ldots, 4\right\}$ [4], which are accompanied by 16 polarization observables [5]. The observables are bilinear hermitean forms in the amplitudes and they divide into the subsets of group $\mathscr{S}$ observables $\left\{\sigma_{0}, \Sigma, T, P\right\}$ and furthermore three classes of beam-target $(\mathscr{B} \mathscr{T})$, beam-recoil $(\mathscr{B} \mathscr{R})$ and target-recoil $(\mathscr{T} \mathscr{R})$ observables with four quantities each. Furthermore, the CGLN-amplitudes can be expanded into a partial wave series defined by electric and magnetic multipoles $\left\{E_{\ell \pm}, M_{\ell_{ \pm}}\right\}$[4].

Once the multipole-series is truncated at $\ell_{\max }$, the observables acquire the following standard-form which is an analog of equation (1):

$$
\begin{gathered}
\check{\Omega}^{\alpha}(W, \theta)=\frac{q}{k} \sum_{n=\beta_{\alpha}}^{2 \ell_{\max }+\beta_{\alpha}+\gamma_{\alpha}}\left(a_{\ell_{\max }}\right)_{n}^{\check{\Omega}^{\alpha}}(W) P_{n}^{\beta_{\alpha}}(\cos \theta), \\
\left(a_{\ell_{\max }}\right)_{k}^{\check{\Omega}^{\alpha}}(W)=\left\langle\mathscr{M}_{\ell_{\max }}(W)\left|\mathscr{C}_{k}^{\check{\Omega}^{\alpha}}\right| \mathscr{M}_{\ell_{\max }}(W)\right\rangle,
\end{gathered}
$$




\begin{tabular}{cc|cc||cc|cc}
\hline \hline & & & & & & \\
Type & $\check{\Omega}^{\alpha}$ & $\beta_{\alpha}$ & $\gamma_{\alpha}$ & Type & $\check{\Omega}^{\alpha}$ & $\beta_{\alpha}$ & $\gamma_{\alpha}$ \\
\hline \multirow{4}{*}{$\mathscr{S}$} & $\sigma_{0}$ & 0 & 0 & & $\check{O}_{x^{\prime}}$ & 1 & 0 \\
& $\check{\Sigma}$ & 2 & -2 & $\mathscr{B} \mathscr{R}$ & $\check{O}_{z^{\prime}}$ & 2 & -1 \\
& $\check{T}$ & 1 & -1 & & $\check{C}_{x^{\prime}}$ & 1 & 0 \\
& $\check{P}$ & 1 & -1 & & $\check{C}_{z^{\prime}}$ & 0 & +1 \\
\hline \multirow{4}{*}{$\mathscr{B} \mathscr{T}$} & $\check{E}$ & 0 & 0 & & $\check{T}_{x^{\prime}}$ & 2 & -1 \\
& $\check{G}$ & 2 & -2 & $\mathscr{T} \mathscr{R}$ & $\check{T}_{z^{\prime}}$ & 1 & 0 \\
& $\check{H}$ & 1 & -1 & & $\check{L}_{x^{\prime}}$ & 1 & 0 \\
& $\check{F}$ & 1 & -1 & & $\check{L}_{z^{\prime}}$ & 0 & +1 \\
\hline \hline
\end{tabular}

Table 1: The parameters listed here describe the angular parametrizations for the 16 polarization observables given in equations (2), (3). The Table and numbers are taken over (up to slight modifications) from Tiator [8].

where we have adopted a notation by Chiang and Tabakin [6] to denote the observables $\check{\Omega}^{\alpha}$ and the index $\alpha$ runs as $\alpha=1, \ldots, 16$. The multipoles present in a certain truncation are sorted in the vector

$$
\left|\mathscr{M}_{\ell_{\max }}\right\rangle=\left[E_{0+}, E_{1+}, M_{1+}, M_{1-}, E_{2+}, E_{2-}, M_{2+}, M_{2-}, \ldots, M_{\ell_{\max }}\right]^{T} .
$$

Table 1 lists a consistent set of parameters defining the photoproduction moment expansion. They are consistent with reference [7], where also the Legendre parametrizations (2) and (3) were published. Tiator [8] showed similarly formalized expansions in the context of a $\cos (\theta)$-series.

The matrices defining the coefficients (3) are hermitean and have dimensions $\left(4 \ell_{\max }\right) \times\left(4 \ell_{\max }\right)$, for $\ell_{\max } \geq 1$. The discrete ambiguities allowed by these bilinear forms have been subject of some studies in the past $[9,10]$. The question whether or not they allow for a unique amplitude extraction in so-called complete experiments has received recent attention [11,12]. In reference [7], we chose to represent these matrices in a color-scheme ('chessboard-plots'), which is exemplified in Table 2.

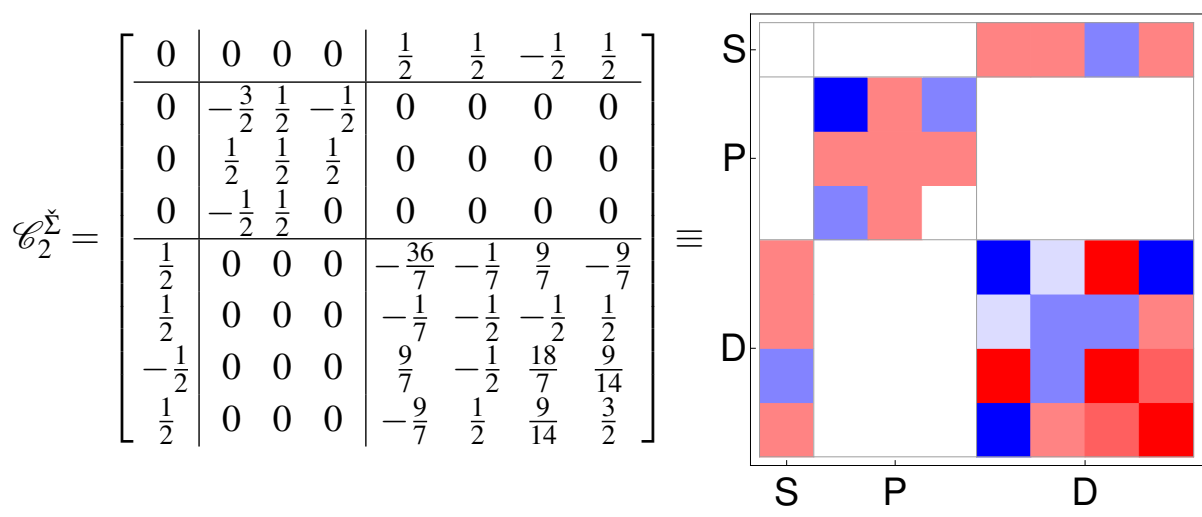

Table 2: The Matrix $\mathscr{C}_{2}^{\check{\Sigma}}$ which defines the coefficient $\left(a_{2}\right)_{2}^{\check{\Sigma}}$ for an expansion of $\check{\Sigma}$ up to $\ell_{\max }=2$ is shown. This is an example for the color-scheme mentioned in the main text. Every matrix element corresponds to a particular interference term. Positive matrix elements are drawn in red, negative ones in blue. The relative strength of each block scales with the size (i.e. modulus) of the respective entry. (color online) 
This formalism allows for moment-analyses in photoproduction under two main aspects:

(i) $\ell_{\max }$-analysis: The form of the angular distribution (2) is fitted for different $\ell_{\max }$. The $\chi^{2} / \mathrm{ndf}$ is compared for different fits, starting with the lower one's. In case it is unsatisfactory, one has to increase $\ell_{\max }$. Once a good fit is obtained, the resulting $\ell_{\max }$ gives in a lot of cases already quite a good estimate for the maximal angular momentum detectable in the data. Plots of $\chi^{2} /$ ndf vs. energy show 'bumps' whenever new important contributions come in (see Figure 1, top).

(ii) Model-comparisons: The fitted Legendre coefficients $\left(a_{\ell_{\max }}\right)_{k}^{\check{\Omega}^{\alpha}}$ can be compared to the right hand side of equation (3) evaluated in terms of multipoles $\mathscr{M}_{\ell}$ stemming from a model. One can do these comparisons switching on/off certain model partial waves and thus sometimes obtains valuable information on which partial wave interferences are important (see Table 3).

Figure 1 and Table 3 show results for moment-analyses for a measurement of the beam-asymmetry $\Sigma$ measured by the GRAAL-collaboration [13]. These illustrative results were discussed during the talk and are also a part of reference [7], where also a lot more details, as well as results for more observables, can be found.

\section{References}

[1] A. V. Anisovich, R. Beck, E. Klempt, V. A. Nikonov, A. V. Sarantsev and U. Thoma, Eur. Phys. J. A 48, 15 (2012).

[2] J. E. Bowcock and H. Burkhardt, Rep. Prog. Phys. 38, 1099 (1975).

[3] A. Gersten, Nucl. Phys. B 12, p. 537 (1969).

[4] G. F. Chew, M. L. Goldberger, F. E. Low, and Y. Nambu, Phys. Rev. 106, 1345 (1957).

[5] A. M. Sandorfi, S. Hoblit, H. Kamano, T. -S. H. Lee, J. Phys. G 38, 053001 (2011).

[6] W.-T. Chiang and F. Tabakin, Phys. Rev. C 55, 2054 (1997).

[7] Y. Wunderlich, F. Afzal, A. Thiel and R. Beck, Eur. Phys. J. A 53, no. 5, 86 (2017).

[8] L. Tiator, AIP Conf. Proc. 1432, 162 (2012).

[9] V. Grushin, in "Photoproduction of Pions on Nucleons and Nuclei", Proceedings of the Lebedev Physics Institute Academy of Science of the USSR (Nova Science Publishers, New York and Budapest, 1989), Vol. 186 (1989).

[10] A. S. Omelaenko, Sov. J. Nucl. Phys. 34, 406 (1981).

[11] Y. Wunderlich, R. Beck and L. Tiator, Phys. Rev. C 85, 055203 (2014).

[12] Y. Wunderlich, PhD-thesis, expected in 2018, University of Bonn (2018).

[13] O. Bartalini et al., Eur. Phys. J. A 26, 399 (2005). 


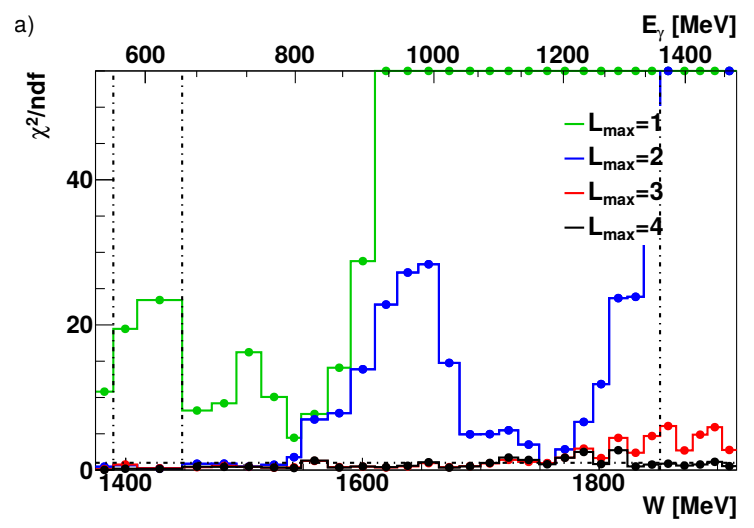

Figure 1: The beam-asymmetry $\check{\Sigma}$ data from the GRAAL-collaboration [13] with only statistical error was fitted using associated Legendre polynomials according to eq. (2) and truncating the expansion at $\ell_{\max }=1, \ldots, 4$. (a) The resulting $\chi^{2} /$ ndf values of the different $\ell_{\max }$-fits as a function of the center of mass energy $W$ and photon LAB-energy $E_{\gamma}$ are shown. (b) 6 out of 31 selected angular distributions of $\check{\Sigma}$ (black points) are plotted together with the different $\ell_{\max }$-fits (solid lines) starting at $W=1504 \mathrm{MeV}$ up to $1885 \mathrm{MeV}$. (c) A comparison of the fit coefficients for $\ell_{\max }=4$ (black points), $\left(a_{4}\right)_{2 \ldots 8}^{\check{\Sigma}_{\text {GRAAL }} \ldots 8}$ (see eq. (2)), with the BnGa2014-02 solution (url: https://pwa.hiskp.uni-bonn.de) truncated at different $\ell_{\max }$ (solid lines) is shown. Colors are the same as in (a). The figures have been published originally in reference [7].

b)
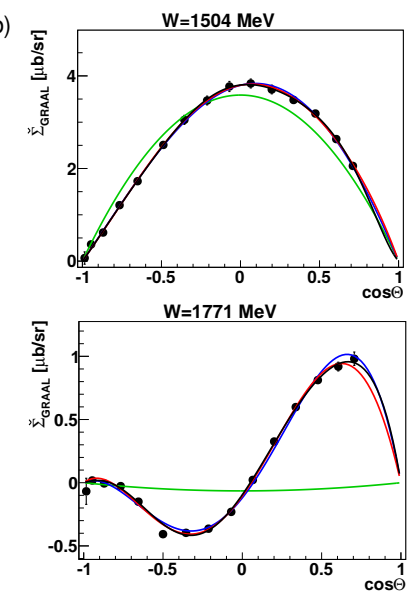

c)
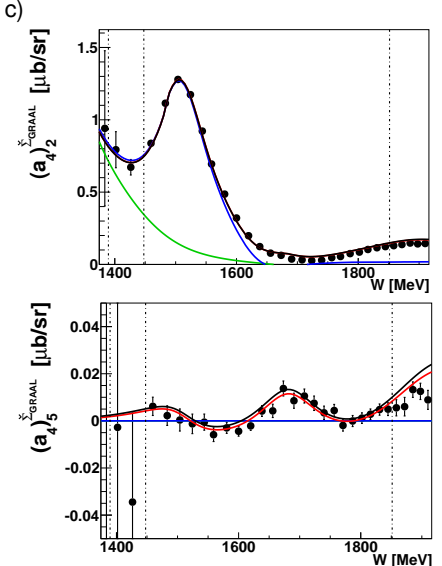
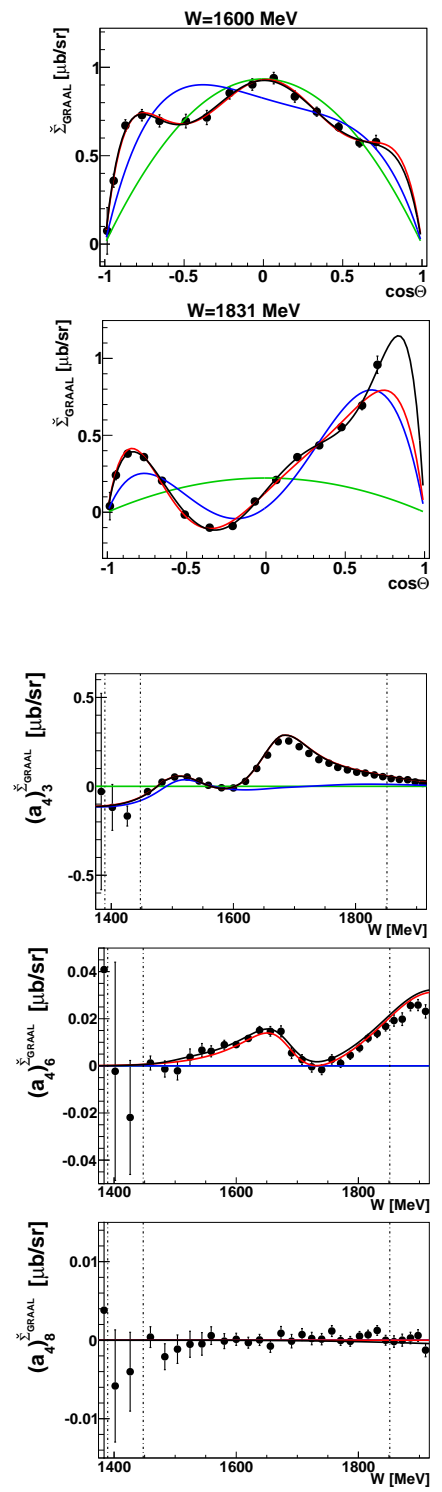
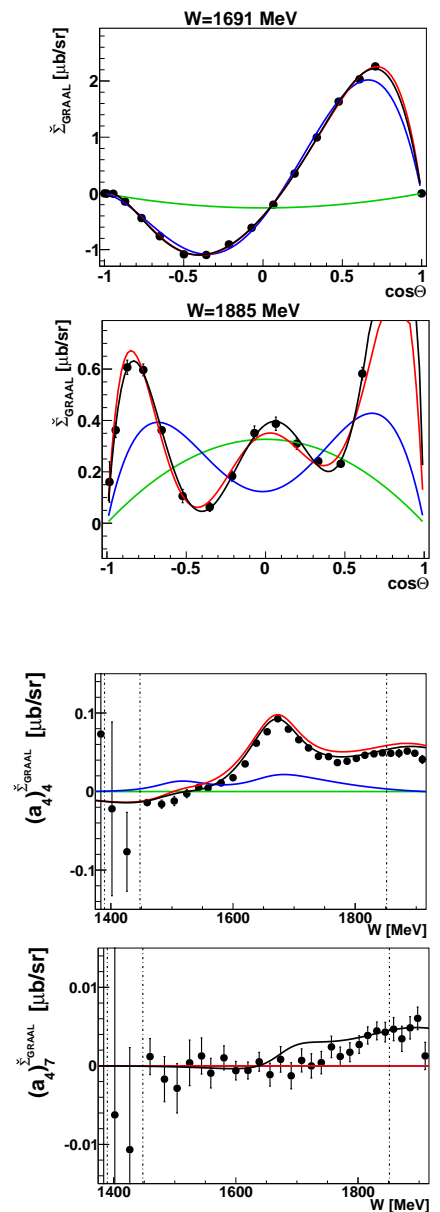

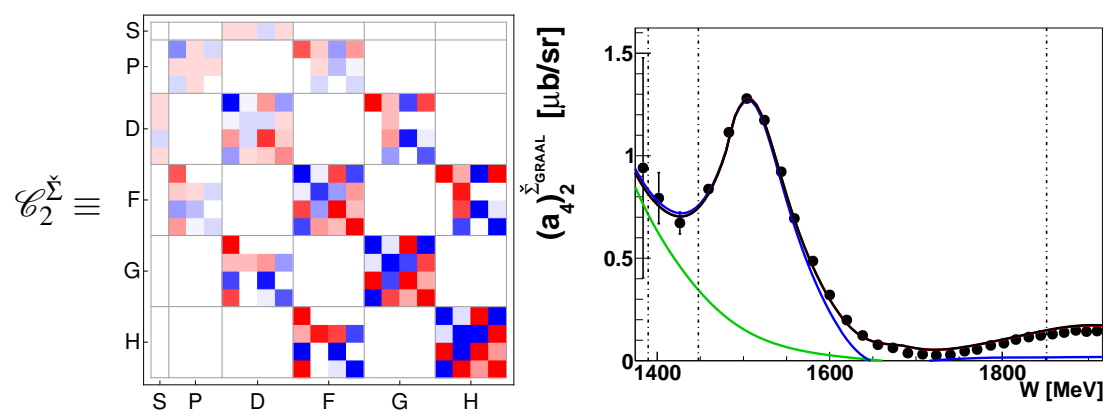

$$
\begin{aligned}
\left(a_{5}\right)_{2}^{\check{\Sigma}}= & \langle S, D\rangle+\langle P, P\rangle \\
& +\langle P, F\rangle+\langle D, D\rangle \\
& +\langle D, G\rangle+\langle F, F\rangle \\
& +\langle F, H\rangle+\langle G, G\rangle \\
& +\langle H, H\rangle
\end{aligned}
$$
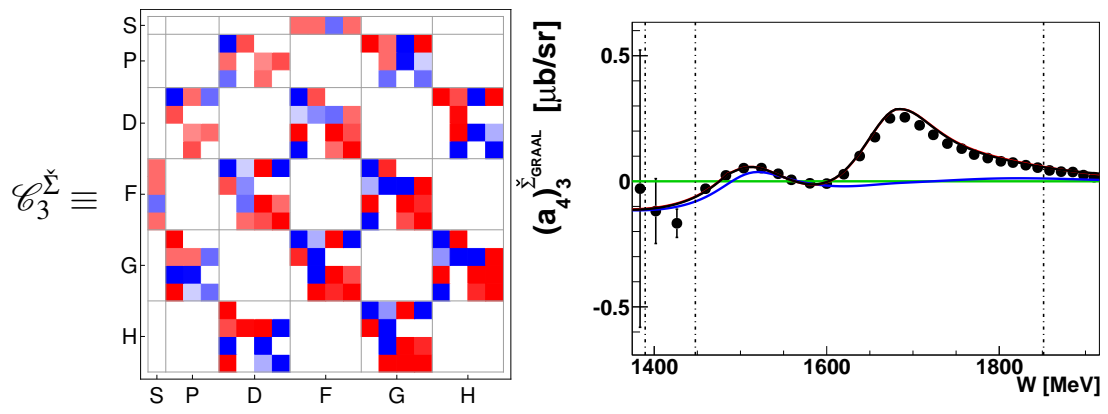

$$
\begin{aligned}
\left(a_{5}\right)_{3}^{\check{\Sigma}}= & \langle S, F\rangle+\langle P, D\rangle \\
& +\langle P, G\rangle+\langle D, F\rangle \\
& +\langle D, H\rangle+\langle F, G\rangle \\
& +\langle G, H\rangle
\end{aligned}
$$
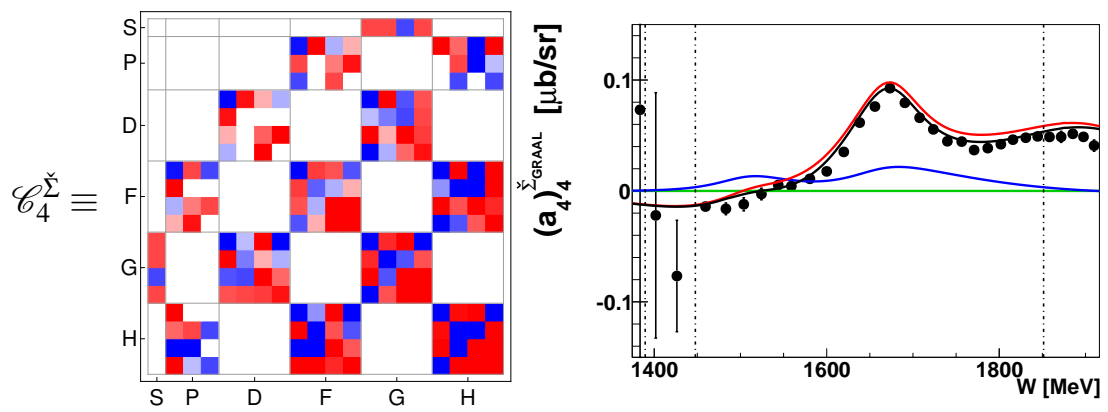

$$
\begin{aligned}
\left(a_{5}\right)_{4}^{\check{\Sigma}}= & \langle S, G\rangle+\langle P, F\rangle \\
& +\langle P, H\rangle+\langle D, D\rangle \\
& +\langle D, G\rangle+\langle F, F\rangle \\
& +\langle F, H\rangle+\langle G, G\rangle \\
& +\langle H, H\rangle
\end{aligned}
$$
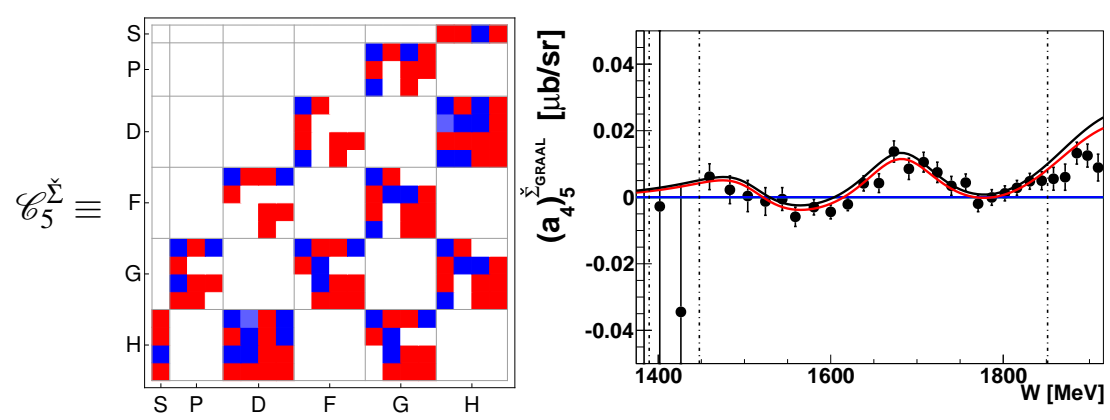

$\left(a_{5}\right)_{5}^{\check{\Sigma}}=\langle S, H\rangle+\langle P, G\rangle$

$+\langle D, F\rangle+\langle D, H\rangle$

$+\langle F, G\rangle+\langle G, H\rangle$

Table 3: Left: The matrices $\mathscr{C}_{2 \ldots 5}^{\check{\Sigma}}$, shown here in the color scheme (cf. Table 2), define the coefficients $\left(a_{5}\right)_{2 \cdots 5}^{\check{\Sigma}}$ for an expansion of the beam-asymmetry $\check{\Sigma}$ up to $\ell_{\max }=5$. Center: Coefficients $\left(a_{4}\right)_{2 \cdots 5}^{\check{\Sigma}_{\text {GRAAL }}}$ obtained from a fit to the $\check{\Sigma}$-data by the GRAAL-collaboration [13] (black points). Coefficients evaluated from BonnGatchina multipoles, truncated at different $\ell_{\max }\left(\ell_{\max }=1\right.$ is drawn in green, $\ell_{\max }=2$ in blue, $\ell_{\max }=3$ in red and $\ell_{\max }=4$ in black) are shown as well. Right: All partial wave interferences for $\ell_{\max }=5$ are indicated ( $S$ means $\ell=1, P$ means $\ell=2, \ldots$ ). These figure have originally been published in exactly this form in reference [7]. We refer the reader to this paper for more details. (color online) 